\title{
From the User's Manual *
}

\section{Main design concepts}

\subsection{Theoretical basis of model}

This chapter introduces the technical side of the ASIR model starting with the general philosophy behind the simulation approach, and then moving on to look at the key statistical assumptions that govern the behaviour of what is probably the most important part of any insurance model - the risk portfolio.

\subsubsection{Discrete and continuous}

There are two basic sets of elements in any simulation model :

a) a set of state variables whose values completely describe the system at any stage - typically, for a financial system, the items in a company's balance sheet and

b) a set of procedures for calculating a new set of values at any stage $\mathrm{N}+1$ from those at stage $\mathrm{N}$ - for a financial system these can be subdivided into :

1. procedures for determining cash flow, and

2. decisions for converting the cash flow into other balance sheet items such as investments.

The definition of "stage $\mathrm{N}$ " in (b) is crucial. We could simulate an insurance company by treating, for example, the receipt of each premium and the payment of each claim as discrete events, and proceed from one event to the next. Since insurance companies work on the principle of the 'law of large numbers', simulation at such a level of detail would be impracticable if it is desired to simulate the state of insurance companies over a time span of several years, rather than days or months. The methodology adopted for this model is therefore basically that of the continuousvariable time-path approach in which the effects of many 'events' are summed (integrated) to give the cash flow over each time-slice, or period. However, a number of simulated discrete events are superimposed on this underlying continuity; these correspond to important, relatively infrequent, discontinuities in the cash flow which significantly affect the state of an insurance company. Examples are : the raising of

* Published in the serie "Etudes et Dossiers", N. 52 and N. 57, November 1981 and June 1982, The Geneva Association. 
capital and payment of dividends, payment of taxes and payment of large claims (chapters 13, 14 and 8 of the User's Manual).

Thus the model is based on aggregative techniques, supplemented by the use of discrete-event simulation where it is thought more appropriate.

\subsubsection{Stochastic and deterministic}

In the main computer simulation, which is designed to be run without userinteraction, the calculating procedures referred to in 1.1.1. must consist of sets of rules, formulated in computer language, which express :

a) the functional dependence of cash flows on current and past values of variables, and on the environment

and

b) the functions transforming cash flow to new values of state variables.

Such sets of rules may consist entirely of algebraic equations. Alternatively, if algebraic equations are combined with conditional statements and random sampling from statistical distributions, they may be probabilistic. A model without any probabilistic rules is completely deterministic; this approach has been used to simulate premium income and expenses, the return on investments, and the management of the investment portfolio. However, the random nature of the risks business requires a stochastic model; so amounts and numbers of claims (and therefore technical loss reserves) are generated by probabilistic rules. In other words, what determines the cash flow generated by payment of claims are statistical distributions, whereas what determines all other cash flows are exogenously determined " fixed" parameters, which includes all decision parameters.

Thus while most rules used in the model are deterministic, the nature of the model is stochastic, since the amounts of claim payments indirectly affect the values of all state variables. Results obtained by running the model should be regarded as indicating a range of possible outcomes (for a given set of exogenous parameters) rather than a definite answer.

\subsection{Structure of the data}

There are two main types of data in the model :

- Input Data ;

- Data generated as the simulation proceeds.

\subsubsection{Input Data}

The input data can be segregated into eight main divisions :

1. General information: comprising the name and description of the simulation run, the random number seed, the numbers of companies, countries and years simulated, the number of simulation periods per year, and some machine dependent output parameters. 
2. Basic country and currency data: comprising names of countries, and names and units of currencies.

3. Basic company data: comprising for each simulated company (except the shadow company) the country of registration, and the initial levels of share capital and premium, current assets and liabilities, and also the number of branches and the country and yearly fixed expenses of each branch ; finally, some machine dependent parameters for any company results produced.

4. Risks and reinsurance data: comprising the number of direct insurance risk classes for each company, including the shadow company, and the parameters specifying the precise nature of each of these classes; the number of reinsurance treaties accepted by each active company, and the parameters specifying them ; also machine dependent parameters for any required detailed results.

5. Reserves and solvency rules data: comprising parameters for the calculation of technical reserves in each country of operation, and parameters for the calculation of the required solvency margin in each country of registration; also parameters for calculating the EEC unit of currency, if required.

6. Investments and investment strategy data: comprising the initial levels and yields of investments for each active company, and the countries where they are held; also the parameters specifying investment strategy, and any data changes during the simulation.

7. Dividends and capital strategy data: comprising capital raising parameters, and dividend payment parameters, for each active company.

8. Operating environment data : comprising inflation and interest rates, exchange rates, and share and property indices for each country; also real growth rates for each risk class, and tax rates for each country.

Appendix A.1 of the User's Manual (here, paragraph 4), which is also the reference guide to the ASIR EDITOR, contains a complete list of the input data required for a simulation. The three-digit item reference number used within the EDITOR always starts with the number of the division in which the data belongs; thus, for example, all risks and reinsurance data are stored in arrays which are given reference numbers between 400 and 499 .

\subsubsection{Simulated Data}

Data generated as the simulation proceeds can be segregated into four main divisions :

S1: Data generated each period from the outcome of the risks business, including numbers and amounts of claims incurred and claims paid, new levels of policies in force, technical reserves and reserve deposits, income from premiums, commissions, expenses, interest payments and claims payments: hence new levels of current assets and liabilities.

S2: Data generated each period from the outcome of investments held, including net income from interest received, new market values of investments, net additions to investments portfolios : hence new balance sheet levels. 
S3: Data generated at end of each simulation year, to form the underwriting revenue account and the profit and loss account of each company, also solvency margin and accounting ratios, i.e. the year's results.

S4: Data parameters updated at the end of each period or other time interval, for example, monetary items such as expenses or average claims which are updated by inflation factors.

As far as possible, only simulated data for the current year is held within the model, and is superseded after the required output of results. Parameters for risks, investments and decisions, however, remain "fixed", and are only altered if updated as in $\mathbf{S} 4$ above or if the CHANGE facility is used. Manual.

The main simulated variables and arrays are listed in Appendix A.2 of the User's

\subsection{Key statistical assumptions}

Central to the operation of any insurance company is the portfolio of insured risks, and this is reflected in the model. At the core of the simulation is a number of direct insurance risk classes, the results of which "drive" the rest of the model. In this section we consider only those classes which contain a (large) number of policies.

In modelling these risk classes there is a fundamental assumption, namely that underlying each risk class is a set of stable probability distributions from which random samples of claims numbers and amounts can be made. ASIR does not attempt to deal directly with joint probability distributions of claims numbers and amounts and times to settlement ; it is difficult to conceptualize such distributions and even more difficult to supply the necessary data parameters. Instead, ASIR generates claims numbers and amounts from marginal distributions, and assumes that the time to settlement is correlated with the size of claim. The data required to specify these distributions has been kept to a minimum; however, users should find this relatively straightforward approach offers a great deal of flexibility, and gives considerable scope for experimentation in specifying different types of risk classes with widely differing characteristics.

Claims modelling is described in detail in Chapter 8 of the User's Manual.

\subsection{Group vs. VLR risks}

In the previous section, we considered risk classes comprising a number of policies having underlying probability distributions of claims numbers and amounts. Risk classes of this type are called GROUP risks and follow the law of large numbers.

It is also possible to simulate VLR risks - very large risks such as the insurance of a Concorde or an oilrig. The characteristics of such risk class are as follows :

1. there is only one policy (i.e. only one item insured);

2. the probability of a claim being incurred is either extremely small or unassessable ;

3. the sums insured are extremely large.

Two alternatives are available for sampling the incurrence of a claim for a VLR : 
1. A very small probability of a claim occurring may be specified - say .0001 . Each period, a simple Bernoulli trial is carried out, with probability $p$ of "success". If this sampling results in a claim, its amount and times of settlement are sampled according to specified distributions (VLR claims may be settled in up to three separate payments). However this method has the disadvantage that many simulation runs may have to be undertaken before such a claim is generated.

2. A claim may be "forced" to occur at a specified time. The amount and settlement times are then sampled as in the first alternative. This "forced occurrence" alternative must be used if it is desired to simulate coinsurance of a VLR by several insurance companies; the VLR (with proportionate premiums and claim amounts) is entered as a separate risk class for each company.

\subsection{Random numbers}

Sampling from statistical distributions in this simulation is carried out by specially designed subroutines which depend only on the generation of a sequence of pseudorandom numbers. Software for this is provided as standard in all modern computer systems, as is the capability of setting different random number seeds if required, which enables different sequences of pseudo-random numbers to be generated in each simulation run. The use of the random number seed to give different modes of operation of the ASIR model is discussed more fully in section 3.2.

There are three major techniques * that can be used for sampling subroutines; which one is actually used for a given distribution depends on the form of the cumulative distribution function and on considerations governing efficient use of the computer. A considerable amount of research has been carried out in recent years in this area, the results of which have been used in the programming of the sampling subroutines.

* Technical note: These techniques are: the inverse transformation method, the envelope rejection method and the composition method. Details can be found in any standard text on simulation (Monte Carlo) methods. 


\section{From the User's Manual}

\section{Preparing data for a simulation}

There are three stages involved in using the ASIR model. In the first stage, the user must enter data appropriate for the particular simulation which is to be carried out. The second stage consists of carrying out the simulation itself, whilst the third stage sees the user studying the results which ASIR has produced. In many cases, examining the results of a simulation run will lead the user to carry out further simulations, and there is typically found a "cycle of use" as depicted in Fig. 2.1 below.

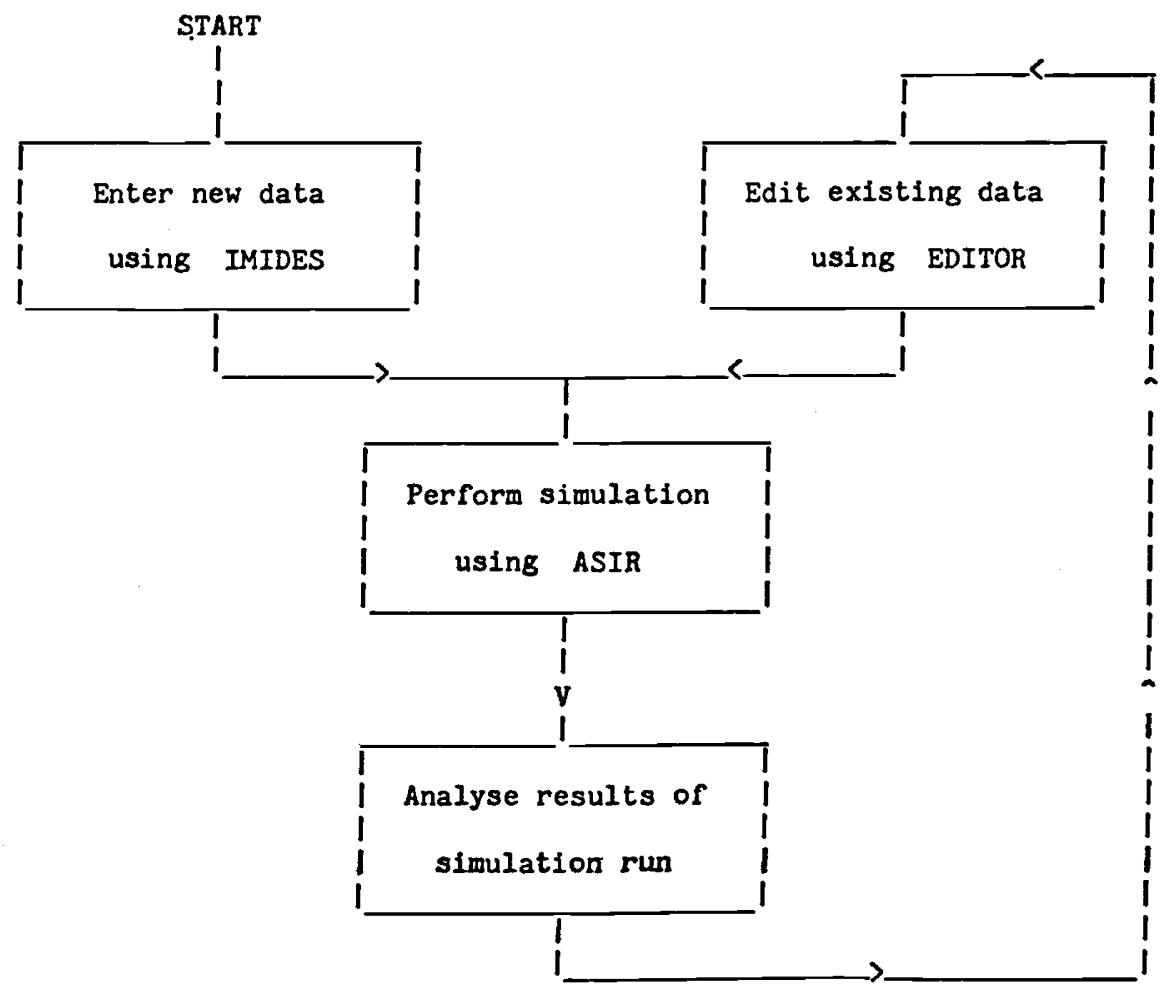

Figure 2.1: ASIR: the Cycle of use 
This chapter is concerned with the first stage : entering data for a simulation. The next chapter explains how a user performes a simulation once the data has been entered.

There are two purpose-built interactive systems which help the user with the task of supplying data for a simulation. These systems are called IMIDES and EDITOR. IMIDES (standing for Insurance Model Interactive Data Entry System) provides a fully prompted "intelligent" data entry and verification system, storing the coded data in computer files which reside on the users' own disc area. EDITOR enables a user to inspect and change any data item stored in any of these files and, when the editing session is complete, EDITOR will store the amended data either in a new data file or back in the old one, at the user's behest. The use of these two systems is now explained in the remainder of this chapter.

\subsection{Interactive data entry using the IMIDES system}

The data requirement for the ASIR model is comprehensive, and many of the data items provide multiple specifications and are linked to other data items. Were it not for the IMIDES system, the task of supplying data for a simulation run could be a formidable one.

IMIDES has been specially designed to ease the task of the user in supplying all the data necessary for a simulation run. It does this by carrying out a " dialogue " with the user in the form of a careful structured question-and-answer session. IMIDES will provide a descriptive prompt for each and every component of data which is needed for a particular simulation run, and will invite the user for his response. This response will be checked against the range of permissible answers and, if correct, will be accepted and IMIDES will then move on to the next question. If the answer was not acceptable, IMIDES will print an explanatory message and invite the user to enter another response.

IMIDES provides the user with an "intelligent" system, because the questions asked will depend on the answers to previous questions. For example, if the user has specified that a particular risk class has two reinsurance treaties, IMIDES will only ask the user to enter details for two treaties for that risk class. As the user enters details for each treaty, IMIDES will check what type of treaty is specified, and will ask only those questions relevant to that type of reinsurance business.

As an additional aid to the user, IMIDES will provide for most of the items of data a default answer, and this will be printed, enclosed in square brackets, as part of the prompt. If the user is willing to accept the default, he needs only to press the RETURN key on the keyboard instead of having to enter the number in full.

A further feature that can assist the user is the "help" facility which will explain the possible answers to questions requiring a coded "key" as the response, e.g. the key to the type of loss reserve estimation.

To start IMIDES, the user must be logged in to the timesharing computing system and should type the command :

\section{RUN IMIDES}


on the terminal. IMIDES will respond with an introductory message followed by the prompt :

Do you wish to create a complete set of data $(\mathrm{Y}, \mathrm{N})$ ? [Y]

IMIDES organises the total set of data into the eight sections outlined earlier:

1. general information ;

2. basic country and currency data ;

3. basic company data ;

4. risks and reinsurance data ;

5. reserves and solvency rules data ;

6. investments and investment strategy data ;

7. dividends and capital strategy data;

8. operating environment data.

These sections are grouped further to obtain three divisions :

COMPANY DATA
ENVIRONMENT DATA
RISKS DATA

sections $1,3,6$ and 7

sections $1,2,5$ and 8

sections 1 and 4

As shown above, the first question put by IMIDES asks the user whether he wishes to enter the complete set of data, or whether he wants to enter just one or two divisions. At the end of the prompt is the letter $Y$ in square brackets, which indicates that the default assumed by IMIDES, unless told otherwise, is " $\mathrm{Y}$ ".

If the user responds with " $N$ ", IMIDES will ask in turn whether the user wants to enter company data, environment data, or risks data. The user can specify any one, two, or even all three divisions.

The next question is :

All data to be placed in one file $(\mathrm{Y}, \mathrm{N})$ ? [Y]

If the user answers " $\mathrm{Y}$ ", then all the data as a result of the session will be placed in a single data file. The alternative response " $N$ " will place different divisions in different files. The second response is preferred if the user wants to create a well organised library of files. In this case the user could create and maintain a number of files containing specifications of risks portfolios. A simulation could then be carried out using any compatible selection from this library. Creating a user library in this way is explained in the next chapter.

IMIDES will next ask for the names of the file or files in which the data is to be stored. Any name is acceptable provided that it is consistent with the requirements of the computer's operating system for the naming of files. (For example, the DECsystem-10 series of computers allows a maximum of six characters in a file name, and these characters can only be letters or digits.) When selecting names, users should keep track of the file names already used, because IMIDES does not check to see whether a name has been used previously and will overwrite any existing files which have the same name. 
Once this initial dialogue is completed, IMIDES will move on to ask for the data to be used in the particular simulation run. Whatever divisions of data have been selected for data entry, IMIDES will always start with section 1 . In this section the user should specify the following items :

1. Name of simulation run - this name is limited to four characters only, and can be used as a short identification. It will appear at the start of any results printed out by the model. The name is stored internally within all the data files produced by IMIDES and does not have to be the same as the name of the file itself.

2. Simulation Description - this can be up to 48 characters long and provides a less restricted way for the user to label a data file. The simulation description also appears at the beginning of any printed listings produced by the ASIR system.

3. Number of active companies - this specifies the number of active companies within the simulation, and must not exceed nine. The shadow company is not included within this total.

4. Number of countries and currencies - this specifies the maximum number of countries and currencies within a simulation.

5. Length of simulation run in years - this determines the maximum length of a simulation run, and can be set to any number required. The computer costs incurred in running a simulation run will be proportional to the length of the simulation.

6. Number of simulation periods per year - the basic accounting unit is the year, but simulations can be carried in smaller time steps of six months or three months, if necessary.

7. Random number seed - this provides the "seed" for the random number generator used by the main simulation program. If the number entered here is zero or positive, that number will be used directly as the seed, and a repeatable set of random numbers will be produced each time the simulation is run. If a negative number is used, then the random number generator will be seeded by looking up the time of day when the simulation is run. This will therefore produce a different set of random numbers each time the simulation is run. However, in both cases, the actual seed used is printed at the beginning of the results listing. The different modes of operation obtainable from the ASIR model through the use of random number seeds is explored further in the next chapter.

8. Default output channels for results - the ASIR model needs to know where output from a simulation is to go, so that it can inform the computer's operating system. In this section, there are three channel numbers which can be specified, although IMIDES will insert default values if the user responds with " $\mathrm{Y}$ " to this question.

The three channel members required are :

i) error messages (default on DECsystem-10 is 5 - the terminal) ;

ii) lineprinter output (default on DECsystem-10 is 3) ;

ii) base number for temporary files used in ASIRRG, or reinsurers' results in ASIR (default on DECsystem-10 is 30). At later stages within IMIDES, the user will also be asked to specify which channel numbers are to be used for the various reports producted in the course of a simulation. 
IMIDES will now proceed to go through each of the data sections requested by the user at the beginning of the session. For each component of data, IMIDES will provide a fully descriptive prompt, and will in most cases provide a default answer indicated within square brackets. IMIDES will then await the user's response. When this is forthcoming, it will be checked for consistency before IMIDES moves on to the next question. If the answer is incorrect, IMIDES will print an error message of the form :

$$
\text { [? Invalid answer - response should lie in range : } 1 \text { - 9] }
$$

and will repeat the original question until the answer provided by the user is within the limits it expects. Sometimes these limits are fixed, e.g. the maximum number of countries/currencies is always 20 ; sometimes they depend on the answer to previous questions, e.g. if the user has indicated a maximum of three countries / currencies, not more than three investment classes can be entered for each active company.

Where ASIR requires a single data item to encompass a number of component data items (e.g. IRISK $(6, N)$ which gives as a three digit code the number of treaties for risk class $\mathrm{N}$ and the type of treaty for each), IMIDES will either ask an explicit series of questions or will deduce the values implicitly from other information it has. In either case, the data item will be filled in automatically without the user having to intervene.

Within the broad grouping of data into divisions and sections, IMIDES also subdivides the data during entry into "checkpoint blocks". At the beginning of each such block, IMIDES prints a message like :

$$
\text { [checkpoint block } 1.1 \text { starts] }
$$

The user will then be asked for a group of data items, and then a message like :

[checkpoint block 1.1 finish] $\rightarrow$ your command (type $h$ for help) ?

will appear. There are four valid responses to this prompt :

1. " $P$ " or just RETURN - this instructs IMIDES to proceed normally to the next block of data to be input. This is the default response.

2. "B" - this instructs IMIDES to backtrack to the beginning of the checkpoint block so that any data entered incorrectly can be entered again. This is especially useful if the user has made a mistake in entering a fundamental item of data such as the number of risk classes insured by each company.

3. "A" - this instructs IMIDES to abort the session immediately. Any data entered up to that point will be lost.

4. " $\mathrm{H}$ " - this instructs IMIDES to print a helpful text explaining the operation of checkpoint blocks. After the text is printed, the prompt shown above will be repeated.

The checkpoint facility thus gives the user an elementary method of editing the data just entered before proceeding further. It is not possible, however, to backtrack more than one checkpoint block.

When all the data in the IMIDES session has been entered satisfactorily, IMIDES will print the message :

*** END OF IMIDES DATA INPUT *** 
and will confirm the creation of the data file or files by printing a message like :

File ENV27 created

for each file named during the initial dialogue. IMIDES will then terminate relinquishing control back to the computer's operating system.

An example of a short IMIDES terminal session is included in Appendix F of the User's Manual.

\subsection{Interactive editing using the EDITOR}

As seen in the preceding section, IMIDES provides the user with a fully prompted system for the entry of a complete division of data, which is subsequently stored in a named data file within the user's own disc area.

The EDITOR is another purpose built interactive system which enables the user to inspect and edit any of the data contained in any of the files. It provides the only effective means of performing this useful task, because the data files used within ASIR contain binary data (they are, in fact, unformatted FORTRAN data files), and standard computer text editors will not make any sense of them.

With the EDITOR a user can check the values of individual data items or columns within an array. After each selected value is displayed, the user has the option to change the value if he should so wish. When an editing session is complete, the user can instruct the EDITOR to overwrite the existing data file with the new data, or the modified data can be saved in a new file.

The EDITOR is particularly useful in the case where a user wishes to run a number of simulations changing a small number of data items in between each run. In this application, the user would create an initial set of data using IMIDES, and from this he could create a series of related files using the EDITOR.

There is one fundamental difference between using IMIDES and using the EDITOR. IMIDES is a fully prompted, "intelligent" system that provides the user with substantial help during the data entry process, and checks to see that all responses made by the user are correct and consistent. It is perfectly possible to use IMIDES without knowing anything about the way in which the data is organised within the ASIR model. The EDITOR, on the other hand, performs virtually no checking whatsoever. Any item of data can be altered to take on virtually any value, even if the new value is inconsistent with other data within the file or is just totally unsuitable. Such errors will only be detected during the simulation, which will abort prematurely with an appropriate error message. When running the EDITOR, the user must therefore ensure that he is making valid alterations to the data. In many cases, the user will need to know something about the way in which ASIR organises data within the simulation arrays ; all this information is given in earlier chapters of the User's Manual and should not present any real difficulties.

There is an exception to the above assertion. Within the EDITOR there is a "super-edit" facility which is invoked whenever a user wishes to deal with the special arrays NBRNCH, IRR, ITRETY, IRV or any of the arrays governing the operating environment. This facility is described further in section 2.2.1. 
To start the EDITOR, the user issues the command :

\section{RUN EDITOR}

After printing an introductory message, the EDITOR will ask :

Name of file to be edited ?

to which the user should respond with the name of the file he wishes to edit. The EDITOR will then load the data from that file into temporary working storage, and will then prompt for :

Item Reference ( $=0$ to finish editing) ? [0]

All the data within the EDITOR are referred to initially by a three digit reference number, all of which appear in Appendix A.1. Suppose that a user wishes to alter the data item referring to "delay between commissions due and paid" for one of the risk classes. This is within item 422, as can be seen from Appendix A.1 of the User's Manual (here, paragraph 4), and so the user would respond with 422 to the above prompt. The EDITOR will then ask :

Risk class number ? [0]

so that it can retrieve the information from the correct risk class for this item. As the response to this, the user should give the sequential risk class within the data file. If, for example, the risk class wanted was the second risk class of company 2 , and companies 0 and 1 had three risk classes each, the user would respond with 8 as the number of the risk class.

For some data items (all those stored as two dimensional arrays), there is also a sub-reference number, which the EDITOR will also prompt for if necessary. In this example, the requested item reference 422 has three data items within it, and so the EDITOR will ask :

Sub-reference for item 422 ( $=99$ for all) ? [0]

As can be seen from the appendix, the sub-reference for the particular data item wanted is 3 .

With this information, the EDITOR can pinpoint the single value requested by the user. The EDITOR will retrieve this information, and will print out the value as currently held, for example :

Old value : 0.25

The EDITOR then asks the user whether he wishes to alter this value. If the user responds with " $\mathrm{Y}$ " to the question :

Change $(\mathrm{Y}, \mathrm{N}) ?[\mathrm{~N}]$

the EDITOR prompts for the :

New value?

The EDITOR will store the user's response, and will return to the :

Item Reference ( $=0$ to finish editing) ? [422]

prompt ready for the next item to be edited.

As a time-saver, the EDITOR will always remember the user's last response to a particular question, and will display this as part of the prompt, as shown above. 
If the user simply responds with a RETURN, the EDITOR will substitute the previously entered value as the default. This applies to all the prompts given to select a particular item of data for editing.

One variation to the above dialogue occurs if the user specifies 99 in response to the sub-reference prompt. In this case, the EDITOR will step through all the sub-reference items for the array and column specified. In the above example, the user is dealing with the 8 th column (i.e. the 8th risk class) of the array RISK. This array has three sub-reference values (i.e. it has three rows). If the user enters the value 99 as the sub-reference, the EDITOR will print each row of the array in turn, and await a response. If the user simply enters RETURN, the value for that row remains unchanged. If the user enters a numerical value, this new value will replace the old one for that row. A typical dialogue would appear something like :

$$
\begin{aligned}
& \text { Sub-reference for item } 422 \text { (=99 for all) ? [ 0] } 99 \\
& \text { Sub-reference 1: [ 1.000]? } \\
& \text { Sub-reference 2: [ } 0.500] ? 0.25 \\
& \text { Sub-reference 3: [ } 0.250] \text { ? } \\
& \text { Item reference ( }=0 \text { to finish editing) ? [422] }
\end{aligned}
$$

Here, the user has changed the value of sub-reference 2 (referring to the mean delay between premiums due and premiums received) from 0.5 to 0.25 , but has left the other two values unchanged.

When the user has finished all his edits, he should respond with a zero as the item reference number. The EDITOR will then ask :

Do you wish to overwrite existing file $(\mathrm{Y}, \mathrm{N})$ ? [N]

$A$ " $Y$ " response will replace the disc copy of the file with the new data, which has been held up to this point in temporary working storage. A " $N$ " reply will, however, invoke the further prompt :

Name of new file for data?

allowing the user to create a new named file to hold the edited data.

Once the EDITOR has satisfactorily disposed of the information it holds, it prints a confirmatory message and then asks :

Do you wish to edit another file $(\mathrm{Y}, \mathrm{N})$ ? [N]

$A$ " $Y$ " reply will return the user to the beginning of the EDITOR program, and he will be asked for the name of the next file to be edited. A " $N$ " reply will terminate the EDITOR, which will relinquish control back to the computer's operating system.

\subsubsection{The "Super-Edit" facility}

Most of the data items stand completely by themselves, and can be edited without regard to any other item of data. A few data items are somewhat more important, and affect many other items of data, for example, the IRISK $(6, \mathrm{~N})$ item mentioned above determines how many treaties are associated with a given risk class. If this data item 
is incorrect, the data in the REINS array (reference number 430 ) will be interpreted incorrectly or may be ignored altogether.

There are a handful of items which are fundamental in nature. They are as follows :

- NBRNCH (reference 303)

- IRR (reference 400)

- ITRETY (reference 431)

- IRV (reference 600)

The reason for their special importance is because they control the interpretation of whole groups of arrays.

The IRV array, for example, governs the number of investment classes held by each active company. The data in all the other arrays in the $6 \mathrm{XX}$ series is then organised based on this information. If, to continue the example further, company 1 has two investment classes company 2 has one investment class, and company 3 has two investment classes, then the data in IRV :

$$
\operatorname{IRV}(1)=2 \quad \operatorname{IRV}(2)=1 \quad \operatorname{IRV}(3)=2
$$

will control the interpretation of all the data stored in arrays $610,611,612,613, \ldots$ and so on. The first two columns of these rows will be assigned to company 1 , the third column to company 2 , and columns 4 and 5 to company 3 . If the user were to edit the IRV array and leave the other arrays untouched, the allocation of investment classes between the active companies would be completely altered.

It would be unreasonable to expect a user to edit manually all the arrays affected by a change to one of the four arrays mentioned above. The EDITOR therefore contains a super-edit facility which is invoked whenever these four arrays are selected for editing. This facility first explains the present allocation of branches / risk classes / treaties accepted/investment classes, and then asks whether this is to be changed. The user can increase or decrease the allocation to a particular company, or leave it unchanged. If, in the above example, the number of investment classes for company 2 were increased from 1 to 2 , the super-edit facility would conduct the following:

Item reference number ( $=0$ to finish editing) ? [422] 600

Company? [ 0 ] 2

Company 2 has one investment class [investment class 3]

Change $(\mathrm{Y}, \mathrm{N})$ ? [N] $\mathrm{Y}$

Add or Delete an investment class (A,D) ? A

Which investment class number? 4

EDITOR will change the data in item references

$610,611,612,613,614,620,621,622,623$, also 420,432 and 632

so that investment class numbers $4-5$ become 5 - 6 
OK to proceed $(\mathrm{Y}, \mathrm{N})$ ? [Y] $\mathrm{Y}$

REMINDER : You will need to enter data into the above items for the new investment class 4

If the user signified his acceptance, the EDITOR would move all the data as indicated, and would check the contents of the CHANGE array 632, moving any of these references affected by the change as appropriate. As can be appreciated, the super-edit facility provides a powerful enhancement to the capabilities of the EDITOR system.

An extension of the super-edit facility is designed to handle the operating environment data in arrays with reference numbers in the range 800 to 811 . If any of these arrays are selected for editing, the EDITOR will first list the entire environment specification for the particular array and country specified. The user can then edit the data with three options at his disposal :

1. A option - this allows interpolation points to be added. The EDITOR will prompt for the year for which a point is to be added, and for the value of the environmental parameter at that time.

2. M option - this allows the user to modify an existing interpolation point. The EDITOR will prompt for the year to be modified and for new value at that time.

3. D option - this enables existing interpolation points to be deleted. The EDITOR will prompt for the year to be removed.

When editing of that particular environmental specification is complete, the EDITOR will reorganise the environment arrays accordingly, and store the new data. Manual.

An example of a short editing session is given in Appendix $F$ of the User's 


\section{From the User's Manual}

\section{Running the ASIR model}

Once the user has entered the data necessary for a simulation, actually running the model is very straightforward. This chapter describes how the two simulation programs ASIR and ASIRRG are run, and also explains the operation of a utility called LISTER.

\subsection{The simulation programs ASIR and ASIRRG}

There are two main simulation programs within the ASIR model: ASIR and ASIRRG. They perform precisely the same calculations and obtain the same answers, but present their results differently. When a user first starts working with a set of data, it is recommended that the ASIRRG program be used first, in preference to the main simulation program ASIR. This is because ASIRRG is more economical of computer resources, but provides sufficient information to enable the user to correct any errors that might occur in the input data. When the simulation is shown to work satisfactorily, the user can turn to the ASIR program which produces results in far greater detail.

The differences between ASIR and ASIRRG are now explained in the following sections, dealing first with ASIRRG.

\subsubsection{The ASIRRG program}

The ASIRRG program is a summary Report Generator (hence the " $R G$ " in the name). The program is started by issuing the command :

\section{RUN ASIRRG}

ASIRRG will first prompt for :

Name of file containing data?

to which the user should respond with the name of the file in his disc area which contains the data appropriate for the simulation to be carried out. If the data is spread across more than one file, the user should initially respond with the name of the first file. ASIRRG will load this file, discover that it only has part of the data it requires, and will repeat the file name prompt. This process will repeat for two or three times until a complete set of data is loaded. 
ASIRRG will then proceed to perform the simulation, and will terminate when the specified number of years has elapsed, or if one of the companies goes insolvent, whichever occurs first. If any errors arise during the execution of the job, a message will be output to the output channel selected by the data item MD (reference number 110), which is normally allocated to the user's terminal. (The IMIDES system will enter a default value of 5 for this channel.) Error messages produced by ASIRRG are listed and explained in Appendix $\mathrm{E}$ of the User's Manual.

During the execution of a job, ASIRRG also makes use of work files allocated to a range of channels in the sequence MD2 $+1, \mathrm{MD} 2+2, \ldots, \mathrm{MD} 2+\mathrm{NC}$, where MD2 is a base channel supplied within the input data (reference number 112), and $\mathrm{NC}$ is the number of active companies. (The IMIDES system will enter a default value of 30 for this base number.)

The results from the ASIRRG program are produced as a time series report listing comprising :

1. ratio measures of underwriting performance, both gross and net of reinsurance ;

2. a listing of some two dozen key measures of underwriting and total company performance encompassing: premiums, claims, underwriting revenue, profit and loss account, capital issues, balance sheet, and solvency.

This record is output from the ASIRRG program on the output channel selected by the data item MD1 (reference number 111), which is normally allocated to the computer system's line printer. However, any suitable device available to the operating system can be selected. (The IMIDES system will enter a default value of 3 for this channel.)

When ASIRRG has completed the output of its results, it relinquishes control back to the computer's operating system.

An example of the output from the ASIRRG program is given in Appendix $F$ of the User's Manual.

\subsubsection{The ASIR program}

When the user wishes to obtain more detailed results from the ASIR model, the main simulation program, ASIR itself, should be run. To start ASIR, the user should enter the command :

\section{RUN ASIR}

The ASIR program will prompt for the :

Name of file containing data?

in the same manner as that for ASIRRG and, when it has all the data, the simulation itself will start. As with ASIRRG, any error messages that occur will be sent to the channel selected by data item MD.

There is a wide range of output reports available from the ASIR program, and these are grouped into five categories:

1. Claims Settlement Analyses - these give, for each risk class, a detailed breakdown of the claims settlement run-off arising from each underwriting year. The printing 
of this report is controlled by the ICSA array (reference number 440); a non-zero entry in this array causes ASIR to produce a Claims Settlement Analysis report for that risk class, which will be output on the channel number specified by that entry. (IMIDES suggests a default value of 22 for each element of the ICSA array if this report is requested. However, it is quite permissible to allocate different channel numbers to different risk classes if the user wants to have the results from different risk classes printed completely separately.)

2. Detailed Underwriting Reports - these give, for each risk class, a statement of the underwriting performance for each year of the simulation. This statement, subdivided into operating periods if this has been specified by the user, provides figures both gross and net of reinsurance, giving details of the operation of any treaties that may be in force for that risk class. A summary revenue account is also provided. The printing of this report is controlled by the IDET array (reference number 441), which operates in the same way as the ICSA array above. (IMIDES suggests a default value of 23 in this case.)

3. Summary Company Results - these give, for each active company, a statement of the aggregate underwriting performance, a profit and loss account, and a summary balance sheet. The MD3 array (reference number 330 ) controls the printing of this report. (IMIDES suggests a default value of 20 for this report.)

4. Detailed Company Results - these give, for each active company, a detailed balance sheet, a statement of unrealized gains and losses on the investments portfolio, and a sources and uses of fund statement. The printing of this report is controlled by the IBDET array in the input data (reference number 331). (IMIDES suggests a default value of 21 for this report.)

5. Reinsurers' Reports - the final report produced by the ASIR model gives an underwriting report looked at from the reinsurers' viewpoint. These reports are produced automatically for each active company accepting reinsurance business, and are produced on channels $\mathrm{MD} 2+1, \mathrm{MD} 2+2, \ldots, \mathrm{MD} 2+\mathrm{NC}$ (see 3.1.1 for an explanation of these terms).

As with ASIRRG, the simulation will proceed for as many years as was requested in the input data, unless one of the companies becomes insolvent before this time is reached.

When the simulation is complete and all the reports produced, ASIR will return control back to the computer's operating system.

An example of each of the reports produced by the main ASIR program appears in Appendix F of the User's Manual.

\subsection{Modes of operation}

The random number seed can effectively determine the mode in which the ASIR model operates, and three distinct modes of operation can be obtained. These are discussed briefly in the following sections. 


\subsubsection{Single shot}

In most cases, a user will only be interested in obtaining a single set of results from a simulation run. Here the "single shot" mode is the most appropriate mode to achieve this goal. The user should specify a positive value for the random number seed (reference number 106), and this will generate a single (repeatable) set of random numbers which will be used by ASIR to generate the requisite probability distributions used for sampling claims. This will be satisfactory in most cases, but there will still be a random element in the results. To circumvent this, the user could also ensure that the IRISK $(4, N)$ parameter for each risk class was set to a negative number. This would provide "zero variance" results, forcing each of the probability distribution generators within the model to return the mean of the distribution every time they were sampled.

\subsubsection{Sensitivity analysis}

In more extensive explorations the user may wish to explore the effect on the insurance company(s) of systematic changes in the input data. Put another way, the user may want to ask a range of "what if ...?" questions to see how the modelled company(s) react to different circumstances. In that case, the random number seed should be set to a positive value, so that ASIR will produce the same stream of random numbers every time the model is run. This will enable the user to change one or more of the input data items from one run to the next, and be sure that the changes in the results produced is solely due to the change in the input data, and not because a different set of claims experience was simulated.

In this sensitivity analysis mode, the user can see exactly how sensitive the system being modelled is to a change in input data. Some of the data items may have only a minor effect on the operating results, whilst others may have a devastating effect. In real life, it would be the latter group that should be of most concern to insurance company practitioners.

\subsubsection{Stochastic modelling}

In some circumstances, the user may want to get an idea of the range of possible results that can arise from a given set of input data. This recognises the fact that, just as in real life, there can be many outcomes from a given set of risks. The results from one ASIR simulation run is just one possible outcome, just as the state of the real world at any time is just one of the very many possibilities that could have arisen.

To carry out a range of simulations using the same input data, but with different risk outcomes, the user should enter a negative value for the random number seed. This will instruct ASIR to generate a different random number seed each time a simulation run is performed (the actual seed chosen will be based on the date and time of day that the simulation is performed, to the nearest tenth of a second). At present, the user must perform the requisite number of simulations and aggregate the results manually, but a later release of the model will automate this process. 


\subsection{The LISTER facility}

There is a utility program which enables a user to obtain a neat and comprehensive listing of the entire contents of any data file produced by IMIDES or the EDITOR.

The LISTER is started by issuing the command :

\section{RUN LISTER}

which will then prompt for :

$$
\text { Name of file to be listed (type « } C R \text { »o finish) ? }
$$

The user should respond with the name of the file he wishes to see. The LISTER will then ask :

\section{Output to TTY or LPT (LPT,TTY) ? [LPT]}

If the user wishes the listing to be sent to the computer's line printer, he should respond with "LPT" (or just RETURN). If the listing is to appear on the terminal, the user should respond with "TTY".

The LISTER will then ask :

Section of file to be listed ( $=0$ to finish, $1,2, \ldots, 7,8,=99$ for all) ?

Any single section of data can be listed out, with the section numbers being the same as those used by IMIDES (see 2.1). When the section has been listed, the same prompt will reappear. When the user has finished listing out parts of the file, he should respond with zero, whereupon the LISTER will return to the file name prompt.

Alternatively, the user can ask for the entire file to be listed out by entering 99 as the section number. In this case, after the listing is produced, the LISTER will return to the file name prompt straight away.

When the user has finished with the LISTER, he should type just a RETURN in response to the file name prompt, and the LISTER will return control to the computer's operating system.

An example of LISTER output appears in Appendix F of the User's Manual.

\subsection{Maintaining a user library}

The structure of the ASIR model makes it particularly easy for the user to organise his work in a systematic fashion. IMIDES can produce data in named files, with a separate file for each type of data. The EDITOR can select any type of file for editing, and produce a new file with amended data. Finally, the simulation programs ASIR and ASIRRG can accept any grouping of compatible data files and perform a simulation from the data contained therein.

With this capability, the concept emerges of an ASIR file library for each user. This library will contain a collection of files describing particular companies, particular portfolios of risks to underwrite, and different kinds of environment against which to operate.

The user can supplement the information in his library by adding new files with IMIDES. Existing files can be amended, and new ones generated with the EDITOR. 
A wide range of simulations can then be carried out using this stock of information. The user could, for example, keep a set of standard operating environments in this library. If there was a need to evaluate quickly the working of a new insurance arrangement, the details could be fed in with IMIDES as a new risk class, and this could be tested against the existing company set up and operating environments, both of which would already be on record within the library. As use of the model increased and more information stored, use of the ASIR model would become more and more efficient.

This idea is depicted in Fig. 3.1 below.

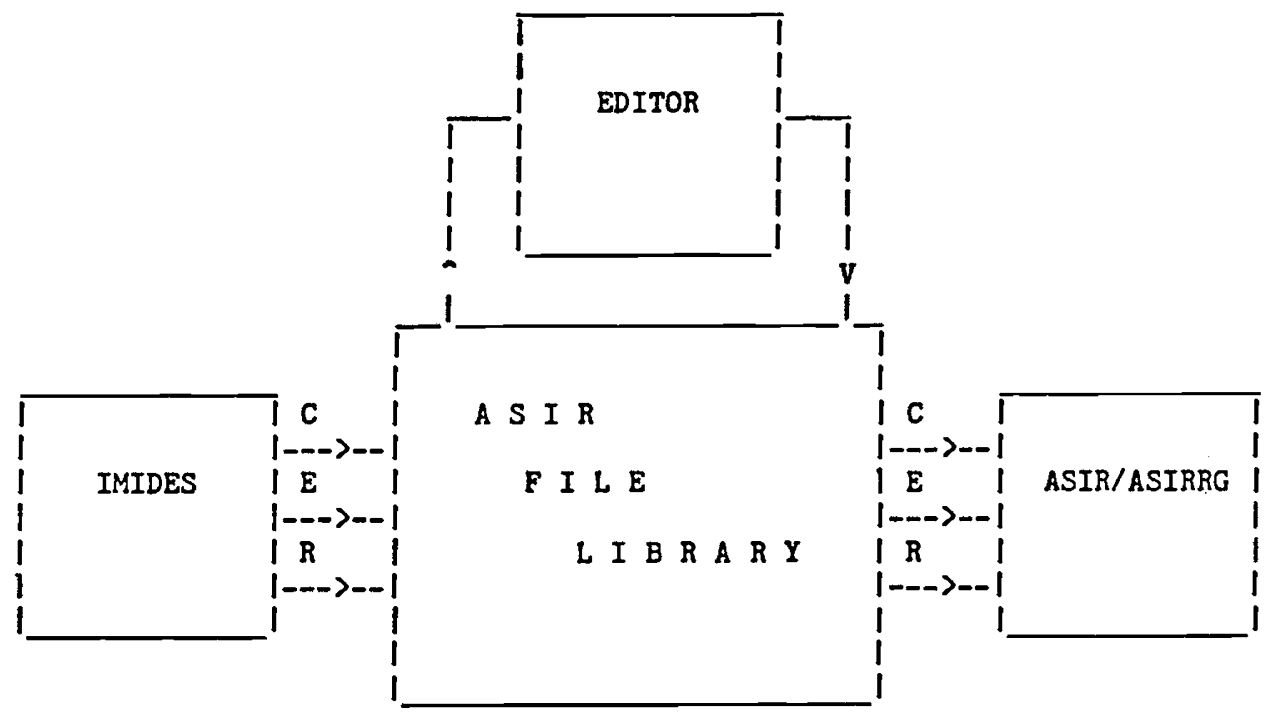

Key : $\mathbf{C}=$ company files

$\mathrm{E}=$ environment files

$\mathbf{R}=$ risk files

Figure 3.1: The ASIR File Library 


\section{From the User's Manual}

\section{Data requirements for ASIR}

4.1. List of input data variables and Editor reference guide

Item Reference

100

101

102

103

104

105

106

110

111

112

200

201

202

203

300

301

302

303

304

310

311

312

313

314

315

320
FORTRAN Variable Description

NAME
NFEAT
IC
KK
YEARS
INT
IRAN
MD
MD1
MD2

KUNTRY $(4,20)$

$\operatorname{KURNCY}(3,20)$

KUNF(20)

KUNL(20)

KHOME(9)

CAPTAL( $(9)$

SHAPRM( $(9)$

NBRNCH( $(9)$

NBKEY(80)

CASH(80)

STERM(80)

ODRAFT(80)

TAXN(80)

DIVID(9)

TAXCRD $(80)$

CEXPNS(80) shadow) only)
Name of simulation run

Description of special features

Number of simulated companies (including

Number of countries and currencies

Length of simulation run in years

Number of simulation periods per year

Random number seed

Output channel number for error messages

Output channel number for lineprinter (ASIRRG

Base channel number for temporary binary files (ASIRRG only)

Country names

Currency names

Units of currency for flows

Units of currency for levels

Country of incorporation of companies

Initial capital

Initial share premium

Number of branches for each company

Country of operation of each branch

Initial level of cash

Initial level of liquid deposits

Initial level of overdraft

Initial level of tax payable

Initial level of dividends declared

Initial level of tax credit

Initial level of "fixed" expenses 
Item Reference

330

331

400

401

410

411

411-1

411-2

411-3

411-4

412

412-1

412-2

420

420-1

$420-2$

420-3

420-4

420-5

$420-6$

420-7

$420-8$

420-9

421 RISKP $(14,40)$

421-1

421-2

421-3

421-4

421-5

421-6

FORTRAN Variable Description

$\operatorname{MD3}(9)$

IBDET(9)

$\operatorname{IRR}(10)$

IPOL(40)

PREM(40)

$\operatorname{DECPRM}(4,40)$

$\operatorname{DXCPRM}(6,40)$

$\operatorname{IRISK}(9,40)$

DXCPRM $(6,40)$

Parameters for linking premium rates to claims
experience

Integer risk class parameters

1st digit: type of business; 2nd digit : key to length of claims runoff

Country where risks taken

Investment class where reserves held

Distributions flag - 1st digit : claims number ; 2nd digit: claims amount

Key to loss reserve estimation

Key to number of treaties and types

Treaty 1 Four digit code AABB

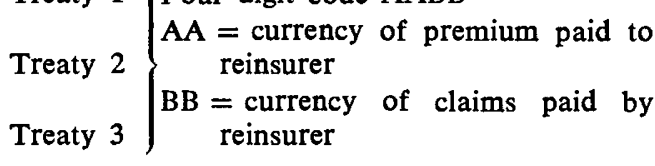

Claims distribution parameters

Group risk

\%age incidence of claims

\%age large claims

Mean size of large claims

SD of large claims

Mean size of small claims

SD of small claims
$V L R$ RISK

Prob. of occurrence / time of $1 \mathrm{st}$ occ.

0.0 / time of second occ. Size of avg. claim

SD of claims

Time occ. to first pmt.

Propn. paid 
Item Reference

421-7

421-8

$421-9$

421-10

421-11

421-12

421-13

421-14

422

422-1

422-2

422-3

423

423-1

423-2

423-3

423-4

423-5

424 RISKN $(4,40)$

424-1

424-2

424-3

424-4

430

$430-1 / 11 / 21$

$430-2 / 12 / 22$

$430-3 / 13 / 23$

$430-4 / 14 / 24$

$430-5 / 15 / 25$

$430-6 / 16 / 26$
FORTRAN Variable Description

Min. time occ. to report Time occ. to scnd pmt.

Mean time ,", , Propn. paid

Min. time occ. to settle Time occ. to third pmt.

Mean time , , , Propn. paid

Max. sum insured ( 0 if open end.)

Max. sum insured or EML

$\left.\begin{array}{l}\text { Least sum insured or EML } \\ \text { Average sum insured or EML }\end{array}\right\}$ For prop. reins.

$\operatorname{RISKT}(4,40)$

Time delay parameters

Time span between changes in premium rates

Mean delay between premium due and premiums received

Delay between commissions due and paid

$\operatorname{RISKE}(5,40)$

Expenses parameters

New business: \%age commissions and expenses

Renewals : \%age commissions and expenses

Fixed expenses

Average expenses per renewable policy

Average expenses per claim reported

Parameters for numbers of policies

$\%$ age renewable policies renewed each period

$\%$ age policies cancelled during term

Optional capacity contraint (sets max. premium to solvency multiple)

EITHER gross premium income in year 1 (if positive)

OR minus number of policies in force at end of year 1 (if negative, and IPOL $=0$ )

OR number of policies in force grows with growth parameters (if zero, and IPOL $>0$ )

REINS $(30,40)$

Reinsurance treaty parameters

1-10 for treaty $1 ; 11-20$ for treaty $2 ; 21-30$ for treaty 3

Reinsurance premium (as \%age of direct premium)

$\%$ age commission

$\%$ age profit commission

\%age of claims paid by reinsurer (QT)

Net retention by ceding company (ST)

Lower limit of net loss covered (EL)

Payment limit on any one risk (QT)

Limit of reinsurers' liability on any one risk (ST)

Upper limit of net loss covered (EL)

\%age cover (EL) 
Item Reference

$430-7 / 17 / 27$

$430-8 / 18 / 28$

$430-9 / 19 / 29$

$430-10 / 20 / 30$

431

432

440

441

450

460

460-1 to 15

$460-42$ to 56

461

461-1

462

$462-1$

$462-3$

$462-4$

462-5
FORTRAN Variable Description

\%age of outstanding losses retained as loss reserve deposits

$\%$ age of premiums retained as UPR deposit $\%$ age interest paid on reserve deposits

\%age of last qtr.'s prem. counted as this qtr.'s ceded prem. (QT or ST)

Deposit premium as \%age of reinsurance premium (EL)

ITRETY(9)

KTRETY(120)

Number of treaties accepted by each company

Keys to treaties accepted

digits 7654321 as follows :

$7 \rightarrow$ treaty 1,2 or 3

$65 \rightarrow$ risk class number 01 to 40

$4 \rightarrow$ ceding company 0 to 9

$3 \rightarrow$ key to treaty type

$21 \rightarrow$ investment class where reinsurer holds reserves

$\operatorname{ICSA}(40)$

IDET(40)

ITYPE(4,10)

Names of types of business

SIMNUM(10,82)

Numbers of outstanding claims reported and settled (risk classes 1 to 10 )

Numbers reported in years 1 to 15

Numbers settled in years 1 to 15

KNOWN(10,5)

Number of claims known outstanding (risk classes 1 to 10 )

Number known outstanding at beginning of simulation

IBNR claims and flags for user specified initial technical reserves (risk classes 1 to 10 )

Number of claims incurred before simulation start but not yet reported

Flag $=999$ for user initialized UPR

Flag $=999$ user specified estimated loss reserves, year 2 onwards

Flag $=999$ for user initialized loss reserve 
Item Reference

463

$463-1$ to 15

464

464-1

$464-2$ to 15

465

$465-1$

470

$470-1$ to 15

471

471-1

474-2 to 15

472

$472-1$

473

473-1

474

474-1
FORTRAN Variable Description

$\operatorname{SIMCLM}(10,41)$

Amounts of outstanding claims gross of reinsurance risk classes 1 to 10)

Amounts of outstanding claims paid in years 1 to 15

$\operatorname{SIMLR}(10,41)$

Estimated gross loss reserve for outstanding claims (risk classes 1 to 10)

Initial loss reserve

Estimates for claims outstanding at beginning of years 2 to 15

Unearned premium reserve (risk classes 1 to 10 ) Initial UPR

$\operatorname{RIMCLM}(30,41)$

Amounts of outstanding claims paid by reinsurers (treaties 1 to 30 covering risk classes 1 to 10) Amounts paid in years 1 to 15

Estimated total claims amounts outstanding payable by reinsurer (on treaties 1 to 30 covering risk classes 1 to 10 )

Reinsurer's initial loss reserve

Estimates for claims payments outstanding at beginning of years 2 to 15

$\operatorname{RIMDLR}(30,5)$

Loss reserve deposits (for treaties 1 to 30 covering risk classes 1 to 10 )

Initial level of loss reserve deposits

RIMUPR(30,5)

Unearned premium reserve of proportional treaty reinsurers (for treaties 1 to 30 covering risk classes 1 to 10 )

Initial level of reinsurer's UPR

$\operatorname{RIMDEP}(30,5)$

Premium reserve deposits, proportional treaties (for treaties 1 to 30 covering risk classes 1 to 10 ) Initial level of premium reserve deposits

Number of bands in user specified claims amount distribution 
Item Reference

481

$481-1$

482

485

486

486-1

487

500

501

501-1

501-2

510

511

520

521

530

531

540

540-1

540-2

540-3

$540-4$

540-5

600

610
ABANDS(16,10)

CUMFAM(15,10)

$\operatorname{NSB}(10)$

SBANDS(11,10)

CUMSET(10,10)

KUPR(20)

$\operatorname{UPR}(2,20)$

$\operatorname{KLR}(20)$

PLR(20)

KRULES(9)

KBAL(9)

KEEC(10)

WEEC(9)

SOLVNT $(5,9)$

IRV(9)

INVEST $(6,40)$
Endpoints of band widths in user specified claims amount distribution

Minimum claim amount

Cumulative percentages of claims (by number) in each amount band

Number of bands in user specified distribution of times between incurrence and settlement

Endpoints of band widths in user specified times to settlement distribution

Minimum time between incurrence and settlement

Cumulative percentages of claims (by number) in each time settlement band

Key to method for calculation of UPR reserve Parameters for UPR calculation Proportional term

Proportion of premium allowable as reinsurance

Key to method for calculation of loss reserve Maximum proportion of reserve allowable as estimated reinsurance gross loss recoveries

Key to solvency margin calculation

Key to valuation of investments

Keys to EEC unit of currency

$\%$ age weighting for each currency used

Solvency calculation parameters

Parameter 1

Parameter 2

Parameter 3

Parameter 4

Parameter 5

Number of investment classes for each company

Integer investment parameters 
Item Reference

610-1

610-2

610-3

610-4

610-5

610-6

611

611-1/5/9

$611-2 / 6 / 10$

$611-3 / 7 / 11$

$611-4 / 8 / 12$

612

$612-1 / 4 / 7$

$612-2 / 5 / 8$

$612-3 / 6 / 9$

613

613-1

613-2

613-3

614

614-1

614-2

614-3

614-4

620

621

621-1

621-2

621-3

621-4

622

622-1

$622-2$

623

623-1

623-2

630
FORTRAN Variable Description

Key to country where investment held

Number of different types of share

Number of different types of bond

Maturity of bond 1

Maturity of bond 2

Maturity of bond 3

PINSHA(12,40)

PINBND $(9,40)$

Parameters for shares

1-4 for share $1 ; 5-8$ for share $2 ; 9-12$ for share 3

Purchase price

Current market value

Gross dividend yield

Beta value

Parameters for bonds

1-3 for bond 1 ; 4-6 for bond $2 ; 7-9$ for bond 3

Purchase price

Coupon

Present value

PINPTY $(3,40)$

Parameters for property

Purchase price

Current market value

Rental income (as \%age of purchase price)

PINCST $(4,40)$

Expenses parameters

\%age expense for share transactions

\%age expense for bond transactions

$\%$ age expense for property transactions

Fixed expense for this investment class

KEYDEC(40)

$\operatorname{DECINV}(4,40)$

Key to investment strategy behaviour

Parameters for overall investment strategy

Parameter for shareholders funds

$\%$ age of total investments as bonds

\%age of total investments as shares

Ratio of current assets to current liabilities

DECBND $(2,40)$

$\operatorname{DECSHA}(2,40)$

NYRCH(31)
Parameters for bonds investment strategy \%age of total bonds as bond type 1 $\%$ age of total bonds as bond type 2

Parameters for shares investment strategy \%age of total shares as share type 1 $\%$ age of total shares as share type 2

Years in which data changes

First element $\rightarrow$ total number of different years in which changes take place Subsequent elements $\rightarrow$ year number for set of changes 
Item Reference

631

632

633

700

701

702

703

800

801

802

803

804

805

806

807

808

809

810

811

815

815-1

815-2

815-3

815-4

815-5

820

820-1

820-2

820-3

820-4
FORTRAN Variable Description

NCHNG(30)

KEYCH(200)

CHNG(200)

NDECYR(9)

DECCAP(9)

DECDIV (9)

DECDFV(9)

$\operatorname{INFLT}(29,20)$

EINFLT $(30,20)$

ISHARE $(29,20)$

SHARES $(30,20)$

INTRST $(29,20)$

$\operatorname{RATE}(30,20)$

$\operatorname{IPROP}(29,20)$

PROPTY $(30,20)$

IGROW $(29,40)$

GROWTH $(30,40)$

$\operatorname{IEXCH}(29,20)$

$\operatorname{EXCH}(30,20)$

$\operatorname{EINTDF}(10,20)$

Interest rate differentials

\%age differential below basic I/R for short term interest rates

\%age differential above basic I/R for overdrafts

\%age differential above basic I/ R for bond type 1

\%age differential above basic I/ R for bond type 2

\%age differential above basic I/ R for bond type 3

Tax rate data

Company profits tax rate (corporation rate)

Imputed tax rate on franked investment income (income tax)

Capital gains tax rate

Miscellaneous taxes rate 